\title{
A scalable hybrid decision system (HDS) for Roman word recognition using ANN SVM: Study case on Malay word recognition \\ ABSTRACT
}

An off-line handwriting recognition (OFHR) system is a computerized system that is capable of intelligently converting human handwritten data extracted from scanned paper documents into an equivalent text format. This paper studies a proposed OFHR for Malaysian bank cheques written in the Malay language. The proposed system comprised of three components, namely a character recognition system (CRS), a hybrid decision system and lexical word classification system. Two types of feature extraction techniques have been used in the system, namely statistical and geometrical. Experiments show that the statistical feature is reliable, accessible and offers results that are more accurate. The CRS in this system was implemented using two individual classifiers, namely an adaptive multilayer feed-forward back-propagation neural network and support vector machine. The results of this study are very promising and could generalize to the entire Malay lexical dictionary in future work toward scaled-up applications.

Keywords: SVM; ANN; Gravity center distance; Hybrid decision system (HDS); Off-line handwriting recognition 